\title{
Wnt signaling pathway is implicated in the alleviating effect of treadmill exercise on maternal separation-induced depression
}

\author{
Jae-Min Lee', Tae-Woon Kim', Sang-Seo Park', Chang-Ju Kim', Mal-Soon Shin², Sam-Jun Lee ${ }^{3}$, Sang-Hoon Kim, ${ }^{1,4}$ Seung-Soo Baek ${ }^{4, *}$ \\ 'Department of Physiology, College of Medicine, Kyung Hee University, Seoul, Korea \\ ${ }^{2}$ School of Global Sport Studies, Korea University, Sejong, Korea \\ ${ }^{3}$ Department of Physical Education, College of Health, Welfare, and Education, Tong Myong University, Busan, Korea \\ ${ }^{4}$ Department of Sport \& Health Care, College of Art \& Culture, Sangmyung University, Seoul, Korea
}

Maternal separation in the developmental stage has a negative influence on brain development and causes depression. The extracellular ligand, Wnt, and its receptors play an important role in axis formation and neural development. Exercise inhibits apoptosis, increases cell proliferation, and exerts antidepressive effect. In this study, the effect of treadmill exercise on the maternal separation-induced depression was investigated in the aspect of Wnt signaling pathway. The maternal separation started on the postnatal day 14 . The rat pups in the exercise groups were forced to run on a treadmill for 30 min once a day from postnatal day 21 to postnatal day 34 . The rat pups in the maternal separation and fluoxetine-treated group were intraperitoneally injected with $5-\mathrm{mg} / \mathrm{kg}$ fluoxetine once a day from postnatal day 21 to postnatal day 34. Forced swimming test was performed to evaluate the depression level. Western blotting was performed for the expressions of Wnt sig- naling ligands, Wnt2 and Wnt3a, and Wnt signaling inhibitors, Dkk1, and sFRP3. Maternal separation showed depressive behaviors in the forced swimming test. Treadmill exercise alleviated depressive behaviors in the maternal separation rat pups. Expressions of Wnt2 and Wnt3a were decreased by maternal separation. Treadmill exercise alleviated maternal separation-induced reduction of Wnt2 and Wnt3a expressions. Expressions of Dkk1 and sFRP3 in the hippocampus were increased by maternal separation. Treadmill exercise alleviated maternal separation-induced reduction of Dkk1 and sFRP3 expressions. Our study demonstrated that treadmill exercise activates Wnt signaling pathway, and then exerted antidepressive effect.

Keywords: Wnt signaling, Depression, Maternal separation, Hippocampus, Exercise

\section{INTRODUCTION}

Maternal separation in the developmental stage has a negative influence on brain development and causes depression (Baek et al., 2012; Marais et al., 2008). Animal model of maternal separation has been used to investigate certain mental disorders, such as depression (Baek et al., 2012; Marais et al., 2008).

The extracellular ligand, Wnt, and its receptors are involved in sign al transduction and they play an important role in axis formation and neural development. Wnt signaling pathway constitutes many signaling molecules and regulates a variety of life phe- nomena by controlling the expression of many genes (Freese et al., 2010). Abnormal regulation of the Wnt signaling pathway is associated with the number of neurological disorders (De Ferrari and Moon, 2006; Inestrosa and Varela-Nallar, 2014). Wnt2 and Wnt3 exert antidepressive effect (Okamoto et al., 2010). Antidepressant treatment increased the expression of Wnt2 and Wnt3 in the hippocampus (Zhou et al., 2016).

Wnt antagonists are divided into 2 functional classes, such as the Dickkopf (Dkk) and the secreted frizzled-related protein (sFRP). Wnt inhibitors, Dkk1 and sFRP3, negatively regulate hippocampal neurogenesis during development. Mice exposed to
${ }^{*}$ Corresponding author: Seung-Soo Baek (D) https://orcid.org/0000-0002-1340-2098 Department of Sport \& Health Care, College of Art \& Culture, Sangmyung University, 20 Hongjimun 2-gil, Jongno-gu, Seoul 03016, Korea

E-mail: ssoop@smu.ac.kr

Received: February 15, 2019 / Accepted: March 21, 2019
This is an Open Access article distributed under the terms of the Creative Commons Attribution Non-Commercial License (http://creativecommons.org/licenses/by-nc/4.0/) which permits unrestricted non-commercial use, distribution, and reproduction in any medium, provided the original work is properly cited. 
mild restraint stress showed increased level of Dickkopf-1, an endogenous inhibitor of Wnt signaling, in the hippocampus (Matrisciano et al., 2011). Deletion of Dickkopf-1 restores neurogenesis and counteracts cognitive decline in old age (Seib et al., 2013). The naturally secreted Wnt signaling inhibitor, sFRP3, is an essential mediator of antidepressive action. Jang et al. (2013b) showed that administration of antidepressant reduced sFRP3 mRNA level, and sFRP3 knockout mice showed a decrease in depressive behaviors.

Exercise inhibits apoptosis, increases cell proliferation, and exerts antidepressive effect (Baek et al., 2012; Cho et al., 2018; Lee and Baek, 2017; Lee et al., 2018). Postnatal treadmill exercise alleviated maternal separation-induced depression by suppressing neuronal cell death and by enhancing cell proliferation in the hippocampus (Baek et al., 2012). Exercise activates the Wnt signaling pathway, leading to improvement of short-term memory (Cho et al., 2018).

In the present study, we investigated the effect of treadmill exercise on the maternal separation-induced depression in the aspect of Wnt signaling pathway. For this study, the expressions of Wnt signaling ligands, Wnt2 and Wnt3a, and Wnt signaling inhibitors, Dkk1 and sFRP3, in the hippocampus were determined.

\section{MATERIALS AND METHODS}

\section{Animals and treatments}

This experiment study was approved by the Institutional Care and Use Committee of Kyung Hee University (KHUASP[SE]16-108). On the postnatal day 14 , the rat pups were divided into one of the five groups: the maternal care group, the maternal care and exercise group, the maternal separation group, and the maternal separation and exercise group, maternal separation and fluoxetine-treated group ( $\mathrm{n}=8$ in each group). The rat pups in the maternal care groups were housed with their respective mothers under the standard conditions, while the rat pups in the maternal separation groups were housed individually. The process of maternal separation started on the postnatal day 14 . The rat pups in the exercise groups were forced to run on a treadmill for 30 min once a day from postnatal day 21 to postnatal day 34 . The exercise load consisted of running at $2 \mathrm{~m} / \mathrm{min}$ for the first $5 \mathrm{~min}$, at a speed of $5 \mathrm{~m} / \mathrm{min}$ for the next $5 \mathrm{~min}$, and then at $8 \mathrm{~m} / \mathrm{min}$ for the last 20 min without inclination. The rat pups in the maternal separation and fluoxetine-treated group were intraperitoneally injected with $5-\mathrm{mg} / \mathrm{kg}$ fluoxetine once a day from postnatal day 21 to postnatal day 34 .

\section{Forced swimming test}

In order to evaluate the depression level of the rat pups, forced swimming test was performed, as previously described method (Cho et al., 2017; Moon et al., 2018). On postpartum day 30, the rat pups conducted a pretest for 15 min to eliminate the acute stress by water and to adapt the animals to the water. Twenty-four hours after the pretest, the rat pups were tested for $6 \mathrm{~min}$. The animals were placed individually into the glass cylinder $20 \mathrm{~cm}$ in diameter and $90 \mathrm{~cm}$ in height, filled with water to a height of 75 $\mathrm{cm}$. The temperature of the water was adjusted to $25^{\circ} \mathrm{C} \pm 1^{\circ} \mathrm{C}$. During the test session, the climbing time and immobility time were analyzed using a Smart version 2.5 video tracking system (Panlab, Barcelona, Spain). Resting was defined when no additional activity was observed other than the actions needed to keep the rat's head above the water. Fasting was defined when the rat was in an active vertical motion with its forelegs above the water level.

\section{Western blot analysis}

Western blotting for the expressions of Wnt2, Wnt3a, Dkk1, and sFRP3 was performed, as previously described method (Cho et al., 2018; Moon et al., 2018). Tissue samples harvested from the hippocampus were lysed in the protein lysis buffer containing $50 \mathrm{mM}$ Tris-HCI (pH, 7.5), $150 \mathrm{mM} \mathrm{NaCl}, 0.5 \%$ deoxycholic acid, $1 \%$ nonidet-P40 (NP40), $0.1 \%$ sodium dodecyl sulfate (SDS), $1 \mathrm{mM}$ phenylmethylsulfonyl fluoride (PMSF), and 100$\mu \mathrm{m} / \mathrm{mL}$ leupeptin. Protein concentration was measured using a colorimetric protein assay kit (Bio-Rad, Hercules, CA, USA). Protein of $40 \mu \mathrm{g}$ was separated on SDS-polyacrylamide gels and transferred onto a nitrocellulose membrane (Schleicher \& Schuell GmbH, Dassel, Germany). Anti-rabbit Wnt2 antibody (1:1,000; Abcam, Cambridge, MA, USA), anti-ribbit Wnt3a antibody (1:1,000; Millipore, Billerica, MA, USA), anti-ribbit Dkk1 antibody (1:1,000; Abcam), and anti-ribbit SFRP3 antibody (1:1,000; LSbio LSBio, Seattle, WA, USA) were used as the primary antibodies. For the secondary antibodies, peroxidase anti-rabbit IgG antibody (1:2,000; Santa Cruz Biotechnology Inc., Santa Cruz, CA, USA) was used. Band detection was performed using as enhanced chemiluminescence detection system (Amersham Pharmacia Biotech GmbH, Freiburg, Germany). Detected bands were calculated densitometrically using Image-Pro Plus software (Media Cybernetics Inc., Silver Spring, MD, USA).

\section{Data analysis}

The results are expressed as mean \pm standard error of the mean. 
IBM SPSS Statistics ver. 23.0 (IBM Co., Armonk, NY, USA) was used for statistical analysis. For the comparison among the groups, one-way analysis of variance and Duncan post boc test were performed with $P<0.05$ as an indication of statistical significance.

\section{RESULTS}

\section{Forced swimming test}

Depression of the rat pups was determined by forced swimming test (Fig. 1). Immobility time was increased and fast time was decreased by maternal separation. Treadmill exercise and fluoxetine treatment decreased immobility time and increased fast time in the maternal separation rat pups.

\section{Wnt2 and Wnt3a expressions}

Wnt2 and Wnt3a expressions are presented in Fig. 2. Expres-
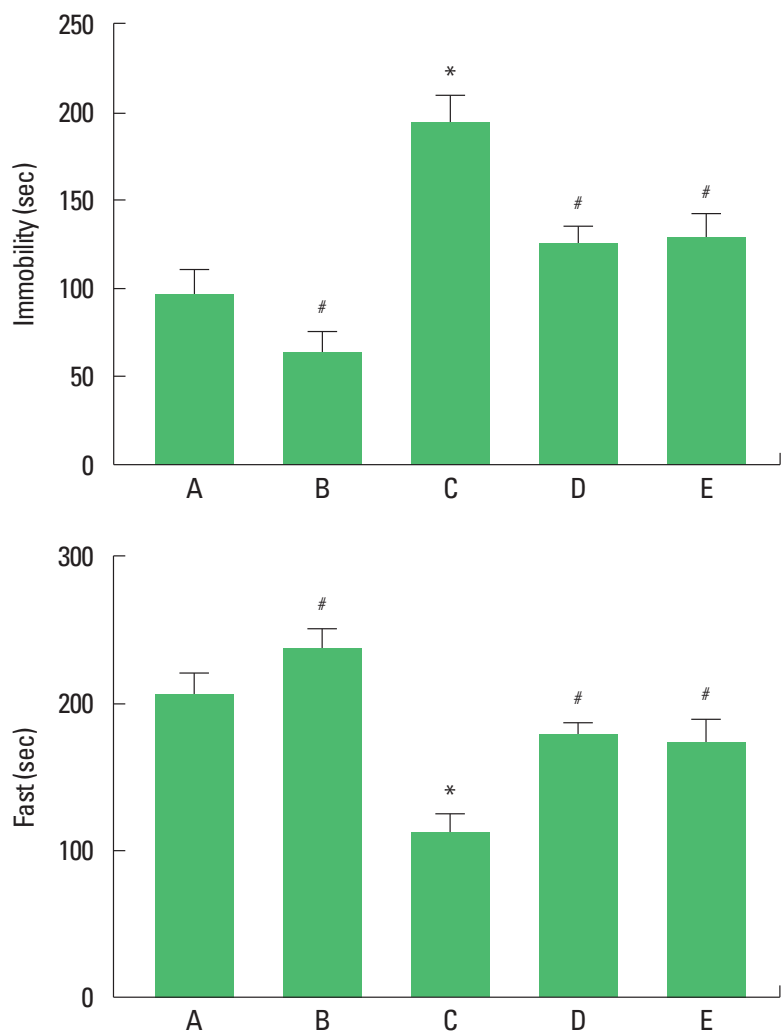

Fig. 1. Immobility time (upper panel) and fast time (lower panel) in the forced swimming test. $A$, maternal care group; $B$, maternal care and exercise group; $C$, maternal separation group; $D$, maternal separation and exercise group; $E$, maternal separation and fluoxetine-treated group. ${ }^{*} P<0.05$ compared to the maternal care group. ${ }^{\sharp} P<0.05$ compared to the maternal separation group. sions of Wnt2 and Wnt3a in the hippocampus were decreased by maternal separation. Treadmill exercise and fluoxetine treatment increased Wnt2 and Wnt3a expressions in the maternal separation rat pups.

\section{Dkk1 and sFRP3 expressions}

Dkk1 and sFRP3 expressions are presented in Fig. 3. Expressions of Dkk1 and sFRP3 in the hippocampus were increased by maternal separation. Treadmill exercise and fluoxetine treatment
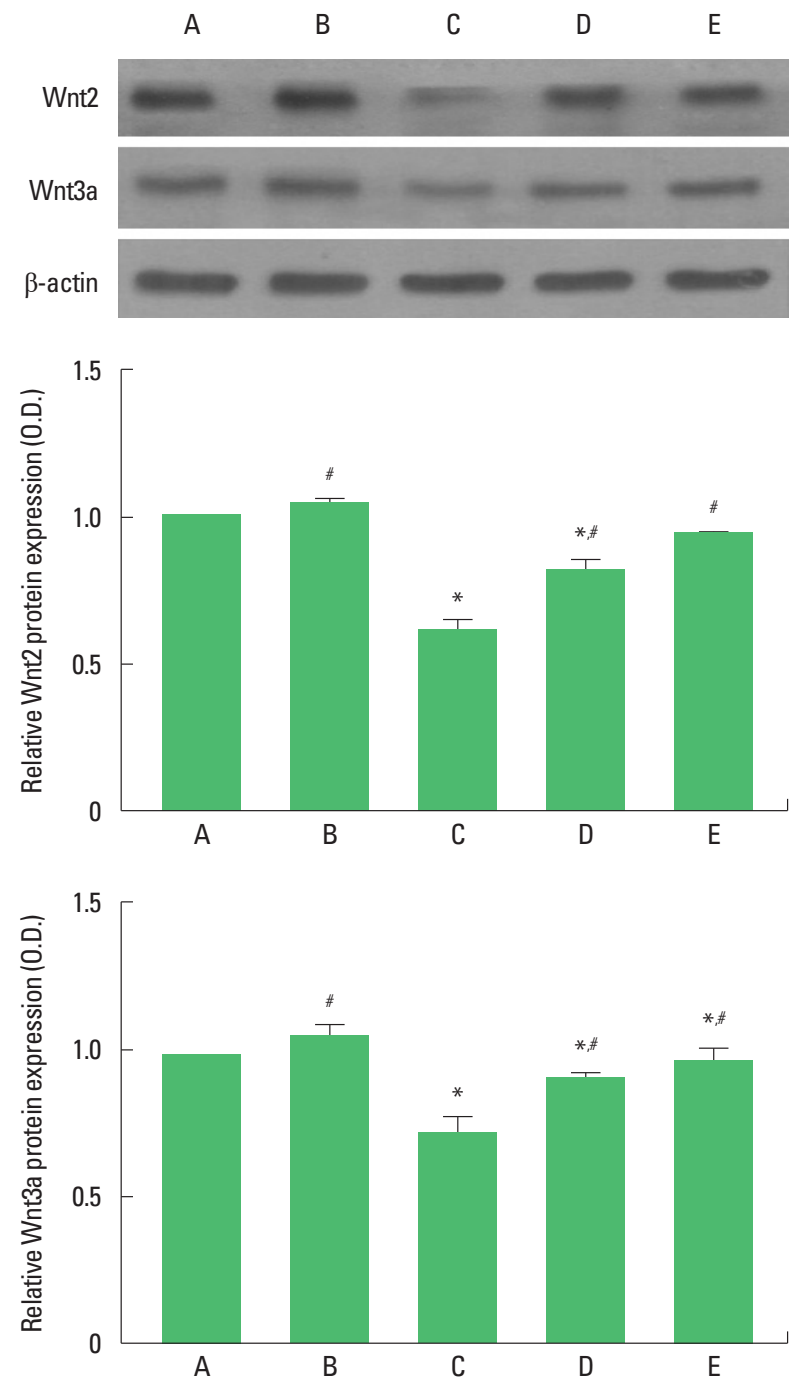

Fig. 2. Upper panel: Presentative expressions of Wnt2 and Wnt3a. Middle panel: The relative expression of Wnt2 in each group. Lower panel: The relative expression of Wnt3a in each group. A, maternal care group; B, maternal care and exercise group; $C$, maternal separation group; $D$, maternal separation and exercise group; $\mathrm{E}$, maternal separation and fluoxetine-treated group. ${ }^{*} P<0.05$ compared to the maternal care group. ${ }^{\#} P<0.05$ compared to the maternal separation group. 

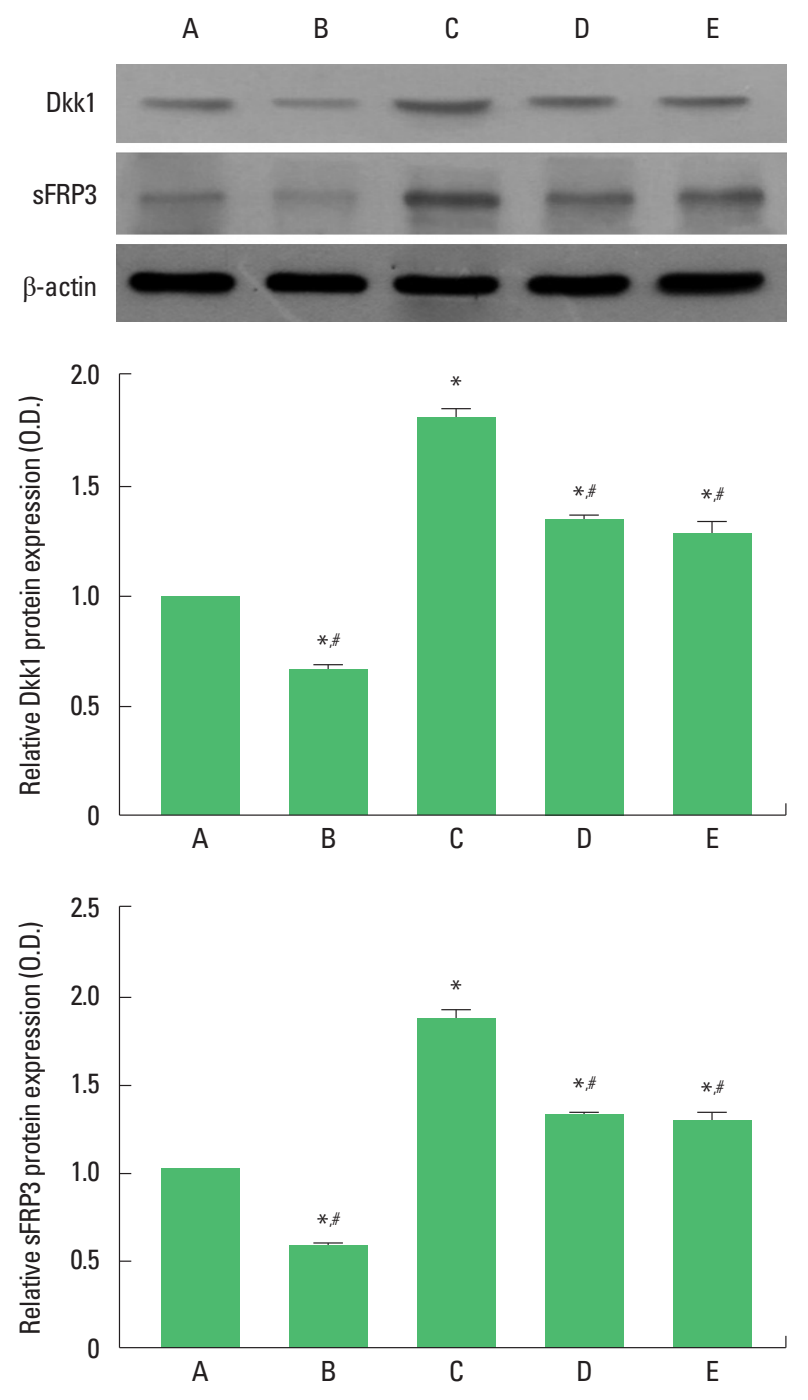

Fig. 3. Upper panel: Presentative expressions of Dkk1 and sFRP3. Middle panel: The relative expression of Dkk1 in each group. Lower panel: The relative expression of sFRP3 in each group. A, maternal care group; $B$, maternal care and exercise group; $C$, maternal separation group; $D$, maternal separation and exercise group; $\mathrm{E}$, maternal separation and fluoxetine-treated group. ${ }^{*} P<0.05$ compared to the maternal care group. ${ }^{\sharp} P<0.05$ compared to the maternal separation group.

decreased Dkk1 and sFRP3 expressions in the maternal separation rat pups.

\section{DISCUSSION}

Maternal separation is known to induce depression-related behaviors (Baek et al., 2012; Marais et al., 2008). Baek et al. (2012) demonstrated that postnatal treadmill exercise or fluoxetine treatment alleviated depressive symptoms by enhancing 5-hydroxy- triptamine (serotonin) and tryptophan hydroxylase expressions in the dorsal raphe. Voluntary exercise reduced depression-like and anxiety-like behaviors in the restrained rats (Lapmanee et al., 2013).

In the present study, maternal separation rat pups showed depressive behaviors in the forced swimming test. However, treadmill exercise alleviated depressive behaviors induced by maternal separation in the rat pups.

Wnt2 expression was upregulated by selective serotonin reuptake inhibitors (Okamoto et al., 2010). Administration of fluoxetine increased Wnt3a expression in the hippocampus (Pinnock et al., 2010). Bayod et al. (2014) showed that the Wnt signaling pathway is activated by moderate exercise. Expressions of Wnt2 and Wnt3 in the hippocampus were suppressed by stress (Zhou et al., 2016). Zhou et al. (2016) demonstrated that knockdown of Wnt2 or Wnt3 in the hippocampus abolished the antidepressant effect of fluoxetine. Treadmill exercise increased Wnt3 expression, and then facilitated neurogenesis in the hippocampus (Kim et al., 2016). Treadmill exercise ameliorated reduction of Wnt3 expression in the cerebral palsy rat pups (Cho et al., 2018).

In the present study, expressions of Wnt 2 and Wnt3a were decreased by maternal separation. However, treadmill exercise alleviated maternal separation-induced reduction of Wnt2 and Wnt3a expressions.

Dkk and sFRP are Wnt inhibitors, and they antagonize Wnt signaling pathway by preventing ligand-receptor interaction or by inhibiting Wnt receptor maturation (Cruciat and Niehrs, 2013). Dkk-1 is a secreted protein that negatively regulates Wnt pathway, and Dkk-1 is related to the process of neurodegeneration (Caraci et al., 2008). Exercise reduced Dkk1 level in the rat hippocampus (Bayod et al., 2014). Jang et al. (2013b) demonstrated that deletion of sFRP3 reduced depression-related behavioral responses in the tail suspension test and forced swimming test. Suppression of sFRP3 activity increased Wnt signaling pathway and then activated neurogenesis (Jang et al., 2013a). sFRP molecules have a more pleiotropic impact on the Wnt signaling cascade, and sFRP is associated with the occurrence and progression of Alzheimer disease (Warrier et al., 2016).

In the present study, expressions of Dkk1 and sFRP3 in the hippocampus were increased by maternal separation. However, treadmill exercise alleviated maternal separation-induced reduction of Dkk1 and sFRP3 expressions.

The present results showed that treadmill exercise ameliorated maternal separation-induced depressive-behaviors. The reducing effect of exercise on depression was achieved through enhancing 
Wnt2 and Wnt3a expressions and suppressing Dkk1 and sFRP3 expressions. Our study demonstrated that treadmill exercise activates Wnt signaling pathway, and then exerted antidepressive effect.

\section{CONFLICT OF INTEREST}

No potential conflict of interest relevant to this article was reported.

\section{ACKNOWLEDGMENTS}

This work was supported by the Ministry of Education of the Republic of Korea and the National Research Foundation of Korea (NRF-2016S1A5A2A01026141).

\section{REFERENCES}

Baek SS, Jun TW, Kim KJ, Shin MS, Kang SY, Kim CJ. Effects of postnatal treadmill exercise on apoptotic neuronal cell death and cell proliferation of maternal-separated rat pups. Brain Dev 2012;34:45-56.

Bayod S, Mennella I, Sanchez-Roige S, Lalanza JF, Escorihuela RM, Camins A, Pallàs M, Canudas AM. Wnt pathway regulation by long-term moderate exercise in rat hippocampus. Brain Res 2014;1543:38-48.

Caraci F, Busceti C, Biagioni F, Aronica E, Mastroiacovo F, Cappuccio I, Battaglia G, Bruno V, Caricasole A, Copani A, Nicoletti F. The Wnt antagonist, Dickkopf-1, as a target for the treatment of neurodegenerative disorders. Neurochem Res 2008;33:2401-2406.

Cho JW, Jung SY, Kim DY, Chung YR, Choi HH, Jeon JW, Han JH. PI3KAkt-Wnt Pathway Is Implicated in exercise-induced improvement of short-term memory in cerebral palsy rats. Int Neurourol J 2018;22 (Suppl 3):S156-164.

Cho JW, Jung SY, Lee SW, Lee SJ, Seo TB, Kim YP, Kim DY. Treadmill exercise ameliorates social isolation-induced depression through neuronal generation in rat pups. J Exerc Rehabil 2017;13:627-633.

Cruciat CM, Niehrs C. Secreted and transmembrane wnt inhibitors and activators. Cold Spring Harb Perspect Biol 2013;5:a015081.

De Ferrari GV, Moon RT. The ups and downs of Wnt signaling in prevalent neurological disorders. Oncogene 2006;25:7545-7553.

Freese JL, Pino D, Pleasure SJ. Wnt signaling in development and disease. Neurobiol Dis 2010;38:148-153.

Inestrosa NC, Varela-Nallar L. Wnt signaling in the nervous system and in Alzheimer's disease. J Mol Cell Biol 2014;6:64-74.

Jang MH, Bonaguidi MA, Kitabatake Y, Sun J, Song J, Kang E, Jun H, Zhong C, Su Y, Guo JU, Wang MX, Sailor KA, Kim JY, Gao Y, Christian KM,
Ming GL, Song H. Secreted frizzled-related protein 3 regulates activity-dependent adult hippocampal neurogenesis. Cell Stem Cell 2013a; 12:215-223.

Jang MH, Kitabatake Y, Kang E, Jun H, Pletnikov MV, Christian KM, Hen R, Lucae S, Binder EB, Song H, Ming GI. Secreted frizzled-related protein 3 (sFRP3) regulates antidepressant responses in mice and humans. Mol Psychiatry 2013b;18:957-958.

Kim DY, Jung SY, Kim K, Kim CJ. Treadmill exercise ameliorates Alzheimer disease-associated memory loss through the Wnt signaling pathway in the streptozotocin-induced diabetic rats. J Exerc Rehabil 2016; 12:276-283

Lapmanee S, Charoenphandhu J, Charoenphandhu N. Beneficial effects of fluoxetine, reboxetine, venlafaxine, and voluntary running exercise in stressed male rats with anxiety- and depression-like behaviors. Behav Brain Res 2013;250:316-325.

Lee HJ, Baek SS. Role of exercise on molecular mechanisms in the regulation of antidepressant effects. J Exerc Rehabil 2017;13:617-620.

Lee JM, Kim TW, Park SS, Han JH, Shin MS, Lim BV, Kim SH, Baek SS, Cho YS, Kim KH. Treadmill exercise improves motor function by suppressing Purkinje cell loss in Parkinson disease rats. Int Neurourol J 2018;22(Suppl 3):S147-155.

Marais L, van Rensburg SJ, van Zyl JM, Stein DJ, Daniels WM. Maternal separation of rat pups increases the risk of developing depressive-like behavior after subsequent chronic stress by altering corticosterone and neurotrophin levels in the hippocampus. Neurosci Res 2008;61: 106-112.

Matrisciano F, Busceti CL, Bucci D, Orlando R, Caruso A, Molinaro G, Cappuccio I, Riozzi B, Gradini R, Motolese M, Caraci F, Copani A, Scaccianoce S, Melchiorri D, Bruno V, Battaglia G, Nicoletti F. Induction of the Wnt antagonist Dickkopf-1 is involved in stress-induced hippocampal damage. PLoS One 2011;6:e16447.

Moon EJ, Ko IG, Kim SE, Jin JJ, Hwang L, Kim CJ, An H, Lee BJ, Yi JW. Dexmedetomidine ameliorates sleep deprivation-induced depressive behaviors in mice. Int Neurourol J 2018;22(Suppl 3):S139-146.

Okamoto H, Voleti B, Banasr M, Sarhan M, Duric V, Girgenti MJ, Dileone RJ, Newton SS, Duman RS. Wnt2 expression and signaling is increased by different classes of antidepressant treatments. Biol Psychiatry 2010; 68:521-527.

Pinnock SB, Blake AM, Platt NJ, Herbert J. The roles of BDNF, pCREB and Wnt3a in the latent period preceding activation of progenitor cell mitosis in the adult dentate gyrus by fluoxetine. PLoS One 2010;5: e13652.

Seib DR, Corsini NS, Ellwanger K, Plaas C, Mateos A, Pitzer C, Niehrs C, Celikel T, Martin-Villalba A. Loss of Dickkopf-1 restores neurogenesis in old age and counteracts cognitive decline. Cell Stem Cell 2013;12: 
204-214.

Warrier S, Marimuthu R, Sekhar S, Bhuvanalakshmi G, Arfuso F, Das AK, Bhonde R, Martins R, Dharmarajan A. sFRP-mediated Wnt sequestration as a potential therapeutic target for Alzheimer's disease. Int J Bio- chem Cell Biol 2016;75:104-111.

Zhou WJ, Xu N, Kong L, Sun SC, Xu XF, Jia MZ, Wang Y, Chen ZY. The antidepressant roles of Wnt2 and Wnt3 in stress-induced depressionlike behaviors. Transl Psychiatry 2016;6:e892. 